\title{
A TEORIA DA MODELAGEM DE DAVID HESTENES NO ENSINO DE CIÊNCIAS E MATEMÁTICA
}

\section{MODELING THEORY OF DAVID HESTENES IN THE SCIENCE AND MATHEMATICS TEACHING}

\author{
Ednilson Sergio Ramalho de Souza \\ Universidade Federal do Oeste do Pará/Instituto de Ciências da \\ Educação/ednilson.souza@ufopa.edu.br
}

\section{Adilson Oliveira do Espírito Santo}

Universidade Federal do Pará/Instituto de Educação Matemática e Científica/adilson@ufpa.br

\begin{abstract}
Resumo
A Teoria da Modelagem considera que as pessoas criam modelos mentais para planejar e para guiar suas interações com o mundo real. Contudo, modelos mentais criados a partir de experiências da vida cotidiana geralmente são incompatíveis com os modelos conceituais da escola. Assim, sustenta-se que o problema fundamental na aprendizagem e na compreensão em ciências e em matemática é coordenar modelos mentais com modelos conceituais. Nosso objetivo é apresentar pressupostos básicos da Teoria da Modelagem, bem como sua aplicação por meio de uma didática conhecida como Modeling Instruction. A questão principal foi saber como são os fundamentos principais dessa teoria e como ela vem sendo aplicada em sala de aula. Trata-se de uma pesquisa bibliográfica com a finalidade de identificar na literatura disponível contribuições sobre o assunto e levantar possibilidade e desafios para o ensino brasileiro de ciências e de matemática. Concluímos que a Teoria da Modelagem tem implicações importantes para a educação científica e matemática.
\end{abstract}

Palavras-chave: Teoria da Modelagem, Modeling Instruction, Ensino de Ciências, Ensino de Matemática.

\section{Abstract}

Modeling Theory believes that people create mental models to plan and guide their interactions with the physical world. However, mental models created from everyday life are generally incompatible with the conceptual models in classrom. Thus, argues that the fundamental problem in learning and understanding in science and mathematics is to coordinate mental models with conceptual models. Our goal is to present the basic assumptions of the Modeling Theory and Modeling Instruction. The main issue was to know how are the main foundations of this theory and how it has been applied in the classroom. This is a literature search to identify contributions to the teaching of science and math. We conclude that the Modeling Theory has important implications for science and mathematics education.

Keywords: Modeling Theory, Modeling Instruction, Scientific Teaching, Mathematical Teaching. 


\section{Introdução}

Para resolver problemas em ciências e em matemática, invariavelmente, os estudantes recorrem a algum tipo de representação simbólica, seja na linguagem oral escrita, na linguagem pictórica, na linguagem matemática. Então esses registros podem, de fato, estar associados a modelos mentais. Não obstante, nem sempre esses modelos mentais podem ser considerados coerentes do ponto de vista científico. Pesquisas mostram que muitos estudantes sabem aplicar corretamente a Segunda Lei de Newton na forma $F=$ ma para calcular a força resultante em um objeto qualquer, mas, devido suas experiências do cotidiano, seguem suas vidas pensando que um objeto só se movimenta se existir alguma força atuando sobre ele (HESTENES, WELLS, SWACKHAMER, 1992; MAZUR, 2015). Então a movimentação per si de inscrições simbólicas parece não ser suficiente para reformular modelos mentais inconsistentes. Por outro lado, a reformulação de modelos mentais pode ser favorecida pelo uso coletivo de múltiplas ferramentas de representação e de comunicação (WELLS, 1987; HESTENES, 2010). É nessa esfera que gira as ideias da Teoria da Modelagem.

A Teoria da Modelagem (TM), desenvolvida nos últimos trinta anos pelo físicoeducador norte americano David Orlin Hestenes, é de natureza cognitiva e procura relacionar modelos conceituais com modelos mentais visando fundamentação pedagógica em ciências e em matemática. Apoia-se na tese principal de que a cognição em ciências, matemática e vida cotidiana é basicamente construção e manipulação de modelos mentais (HESTENES, 2010). Não obstante, modelos mentais gerados na interação com o mundo real geralmente são incompatíveis com modelos conceituais ensinados nas escolas e nas universidades, isso demanda coordená-los de alguma maneira em situação de ensino e de aprendizagem. Assim, a TM foi desenvolvida com recursos da ciência cognitiva, da neurociência, da linguística cognitiva, da pesquisa em ensino de física para investigar implicações da tese acima, resultando em uma didática conhecida como Modeling Instruction.

A Modeling Instruction ${ }^{1}$ foi fruto de um amplo programa de pesquisa desenvolvido desde os anos 1987 por David Hestenes e seus colaboradores. Seu objetivo inicial foi planejar, executar e avaliar atividades didáticas no ensino de física a partir dos pressupostos da TM. Com o sucesso dessa didática, amplamente comprovado por diversas pesquisas ao longo das últimas três décadas (HALLOUN e HESTENES, 1987; WELLS, 1987; WELLS, HESTENES, SWACKHAMER, 1995; DESBIEN 2002; JACKSON, DUKERICH e HESTENES, 2008; BREWE, KRAMER, O'BRIEN, 2009; BREWE, 2008; HEIDEMANN, ARAÚJO e VEIT, 2012; DYE et all., 2013; KILPELA, 2013; DITMORE, 2016), a MI saiu dos muros do ensino de física e, de acordo com a AMTA (2017), tem sido promissora no ensino do chamado STEM (ciência, tecnologia, engenharia e matemática).

Dois métodos complementares destacam-se como importantes na aplicação da Ml: o ciclo de modelagem (WELLS, 1987; SOUZA e ROZAL, 2016) e a gestão do discurso de modelagem (DESBIEN, 2002). No ciclo de modelagem, ocorre tanto o desenvolvimento quanto a aplicação de um modelo conceitual. No discurso de modelagem, o professor

\footnotetext{
${ }^{1}$ No decorrer do texto, usaremos a sigla MI para o termo em língua inglesa Modeling Instruction. Evitamos a tradução para o Português para não correr o risco de significados equivocados.
} 
encaminha situações argumentativas coletivas de modo a fundamentar as conclusões e as afirmações dos estudantes em justificativas e fundamentos científicos, com apoio ostensivo de múltiplas representações simbólicas.

Apesar de importante por apresentar-se como um possível referencial teórico para a educação nacional, a revisão de literatura mostra que a TM não é muito utilizada aqui no Brasil. Assim, a questão norteadora foi saber como são os fundamentos principais dessa teoria e como ela vem sendo aplicada em sala de aula. $O$ objetivo principal é apresentar a TM como possibilidade teórica ao ensino de ciências e de matemática. Especificamente, temos como objetivos: caracterizar pressupostos básicos da TM; analisar sua aplicação em sala de aula; levantar implicações considerando o contexto educacional brasileiro. Trata-se, portanto, de uma pesquisa bibliográfica (MALHEIROS, 2011) com a finalidade de identificar na literatura disponível contribuições sobre o assunto e levantar possibilidades e desafios para a educação brasileira. Resultados preliminares indicam que a TM pode implicar diretamente na prática científica e matemática.

$\mathrm{Na}$ seção que segue, apresentaremos alguns pressupostos que consideramos fundamentais para entender, mesmo que de maneira introdutória, o funcionamento da TM. Para isso, daremos atenção aos conceitos de modelo conceitual e de modelo mental. $\mathrm{Na}$ segunda seção, nossa atenção estará voltada à aplicação da TM nas aulas de ciências e de matemática a partir da didática da Ml. A terceira seção finaliza com nossas considerações e perspectivas de pesquisas considerando o cenário educacional brasileiro.

\section{A Teoria da Modelagem}

A TM é de natureza cognitiva e considera que as pessoas criam modelos mentais para planejar e para guiar suas interações com o mundo real. Apesar disso, pesquisas em educação em ciências (HESTENES, WELLS e SWACKHAMER, 1992; MAZUR, 2015) têm encontrado que modelos mentais criados a partir de experiências da vida real são geralmente incompatíveis com os modelos conceituais apresentados nos livros didáticos. Em função disso, D. Hestenes (2015a) sustenta a tese de que o problema fundamental na aprendizagem e na compreensão em ciências e em matemática é coordenar modelos mentais com modelos conceituais. Assim, a TM foi desenhada com recursos da ciência cognitiva para investigar implicações dessa visão e guiar o desenvolvimento de uma didática e o planejamento curricular. Na presente seção, nosso objetivo é discutir sobre pressupostos fundamentais da TM.

Visando investigar a tese acima, no final dos anos 1980, D. Hestenes começou um programa de pesquisa cuja finalidade foi a aplicação de uma teoria para planejar 0 currículo e o ensino e para avaliar resultados e revisar métodos didáticos. A Figura 01 fornece uma visão geral desse programa. Destacamos que a TM foi desenvolvida ao longo de dois ramos. $O$ da direita, que investiga práticas científicas com modelos conceituais, que são explícitos e observáveis, promovem uma janela para estruturar e processar pensamento científico e o pensamento matemático. E o da esquerda, que investiga a cognição com modelos mentais, que são implícitos e idiossincráticos. Desse modo, modelagem conceitual e modelagem mental são pilares na teoria hestenesiana. 


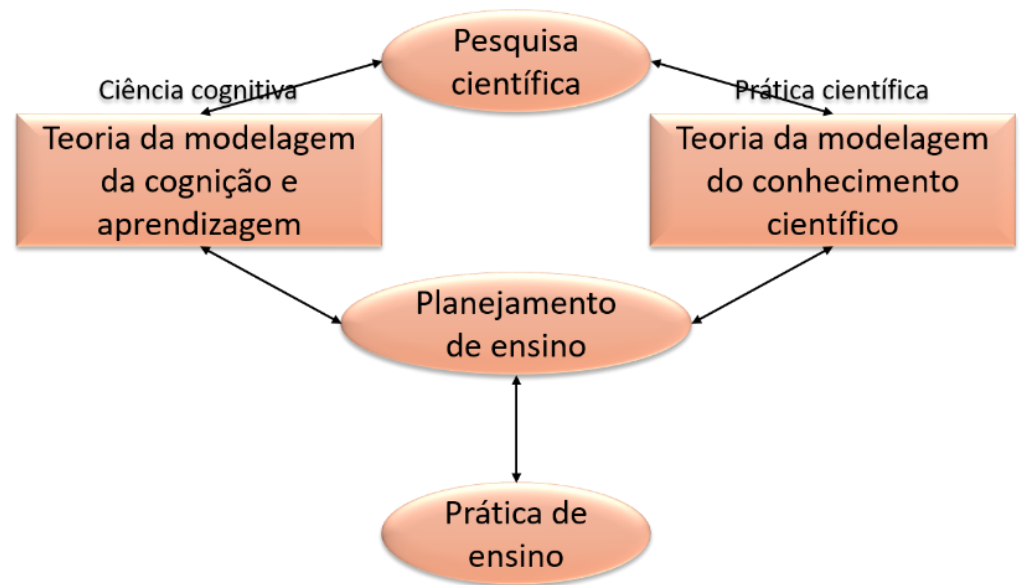

Figura 1 - Teoria da Modelagem: um programa de pesquisa (Fonte: HESTENES, 2010, p. 17, tradução nossa).

Frutos desse programa de pesquisa, dois princípios são propostos como fundamentais na TM. O primeiro estabelece que: "cognição é basicamente construção e manipulação de modelos mentais" (HESTENES, 2010, p. 20, tradução nossa). O autor chama este princípio de Princípio da Primazia dos Modelos. Ressalta que para uma pesquisa guiada por ele o aceitável não é que seja verdadeiro, mas que seja produtivo, isto é, que leve a predições significativas para serem empiricamente testáveis. Tal princípio subtende que a capacidade cognitiva humana para criar, para manipular e para recordar de modelos mentais foi desenvolvida para facilitar a competição com o meio ambiente, então os modelos mentais são centrais para o pensamento e para comunicação. Além disso, o Princípio da Primazia considera que a cultura humana tem ampliado e aumentado sua capacidade de criar sistemas semióticos, ou seja, sistemas de representação por signos (símbolos, diagramas, sinais, ícones etc.), mais notavelmente a linguagem escrita e a linguagem falada. Assim, o conhecimento científico e o conhecimento matemático foram expandidos pelo uso deliberado e autoconsciente de sistemas simbólicos.

O Segundo princípio estabelece que: "modelos mentais possuem cinco tipos básicos de estrutura: sistêmica, geométrica, descritiva, interativa, temporal" (HESTENES, 2010, p. 20, tradução nossa). Este princípio é chamado pelo autor de Princípio das Formas Universais. Comenta o autor que este princípio abastece a TM com um rico sistema de conjecturas para investigar modelos mentais. Além disso, ele serve como um sistema de conceitos básicos envolvidos na caracterização das formas universais.

Assim, a TM é fundamentada na hipótese principal de que a atividade cognitiva primária em ciências e em matemática envolve construção, validação e aplicação de modelos conceituais (HESTENES, 2015b). Percebe-se que dois conceitos surgem como fundamentais no arcabouço da TM: os conceitos de modelo mental e de modelo conceitual, para os quais dirigiremos os holofotes.

\section{Modelos mentais}

A ideia de modelo mental é essencial no arcabouço da TM. O termo modelo mental ganhou força no início dos anos 1980 quando o psicólogo norte-americano Philip 
Johnson-Laird publicou o livro "Mental Models", uma obra pioneira no assunto em que propõe uma teoria para explicar o raciocínio e a compreensão com base em representações mentais analógicas ao mundo real. Ao que parece, por ser contemporânea à $T M$, a teoria dos modelos mentais influenciou grandemente 0 pensamento de David Hestenes. Assim, começar pelo que diz o próprio Johnson-Laird parece ser um bom caminho para refinar a ideia superficial que apresentamos até aqui sobre modelos mentais.

$\mathrm{Na}$ perspectiva da Johnson-Laird: "a percepção produz um modelo mental, a compreensão linguística produz um modelo mental, o pensamento e o raciocínio são manipulações internas de modelos mentais" (JOHNSON-LAIRD, 2005, p. 179, tradução nossa). Quando as pessoas percebem o mundo, sublinha este autor, a visão produz um modelo mental de que as coisas estão acontecendo num cenário a nossa frente. Da mesma maneira, quando as pessoas compreendem a descrição do mundo, elas podem construir uma representação similar desse mundo, embora menos rica: um modelo mental baseado nos significados da descrição e sobre seus conhecimentos prévios.

Uma primeira impressão sobre modelos mentais nos indica que eles não são conhecimentos "soltos", ou seja, sem estrutura sistematizada. Por serem estruturados cognitivamente, os modelos mentais subjazem a percepções, intuições, raciocínios. Desse modo, os modelos mentais estão diretamente relacionados à capacidade de compreensão e de raciocínio do sujeito. São eles que guiam os mecanismos cognitivos que geram explicações, justificativas, deduções. Um bom mecânico de automóvel é capaz de dizer onde está o defeito do motor sem precisar abri-lo. Ele utiliza um modelo mental do motor do carro. A razão nos mostra que o mecânico elaborou seu modelo mental em diferentes momentos de sua profissão, com base em erros e acertos, assimilando cada vez mais informações que pudessem enriquecer o modelo mental e desprezando outras que em nada poderiam contribuir para sua evolução (SOUZA, 2013).

Ou seja, um sujeito começa com modelos mentais simples, que representam apenas aspectos parciais de um fenômeno ou de um sistema considerado real. Esses modelos iniciais são testados e reforçados em diversas ocasiões de sua vida. Os modelos mentais são formados por estruturas que permitem prever e explicar muitas das observações feitas na vida cotidiana de maneira mais ou menos direta, além de facilitar a produção de inscrições simbólicas. Tais modelos podem vir a ser refinados com o tempo, em maior ou menor grau dependendo do envolvimento e interesse do sujeito por mais conhecimentos naquele domínio. Na escola, o conhecimento assimilado interage com os modelos mentais prévios para produzir novos modelos. Dessa interação, surge um sistema mental ainda mais elaborado que contém novas propriedades para descrever e para explicar novos eventos e novas situações. Evidentemente, para que o sujeito possa adquirir modelos mentais próximos aos científicos, é necessária uma série de reformulações cognitivas, resulta que dificilmente isso ocorre em uma única aula, como acreditam muitos professores e gestores da educação.

Assim, para compreender uma situação em face da realidade, o sujeito precisa formar um modelo mental para nortear raciocínios e tomadas de decisão. Consideremos que a situação a ser modelada seja referente ao efeito estufa. Partindo da teoria dos modelos mentais, o sujeito que forma um modelo mental adequado para este sistema é 
capaz de simular mentalmente seu funcionamento para fazer previsões e explicações; pode dizer o que o sistema contém, como ele funciona e por que se comporta de determinada maneira. Um modelo mental consistente é formado conforme o sujeito acrescenta inconscientemente informações a tal modelo, conforme o utiliza para analisar sistemas semelhantes e sistemas diversos. Em função dessas várias modificações no modelo mental inicial, é formado um sistema mental que capacita o sujeito a compreender significativamente o objeto de estudo, podendo transferir tal modelo a novos sistemas e gerar novas aprendizagens.

Entender principais características dos modelos mentais pode ajudar na tarefa de ensino por modelagem mental, algumas características estão resumidas no Quadro 1:

\section{Quadro 1: Principais características dos modelos mentais.}

\begin{tabular}{|l|}
\hline Modelos Mentais \\
\hline São esquemáticos, representam apenas algumas características do sistema modelado. \\
São estruturados sistematicamente, consistindo de elementos e de relações. \\
Seus elementos são normalmente objetos (ou coisas). \\
As propriedades dos objetos são idealizadas (pontos, linhas ou planos). \\
Modelos objeto são sempre colocados no plano de fundo (contexto ou quadro). \\
Objetos individuais são modelados separadamente a partir de um quadro, então eles podem \\
mover-se ao redor de um quadro mental.
\end{tabular}

Fonte: Hestenes (2006, p. 47, tradução nossa).

No contexto da sala de aula, os estudantes chegam com seus modelos mentais prévios formados a partir de seus modos de vida, de suas ocupações, de suas idiossincrasias, de aprendizagens anteriores. Esses modelos retêm percepções e concepções sobre o mundo concebido como real. O professor, acostumado ao método meramente expositivo, apresenta definições, faz exemplos, propõe lista de exercícios como preparação para a prova; dificilmente, valoriza os modelos mentais prévios dos estudantes. Estes, não acomodando modelos mentais cada vez mais inclusivos, não formam modelos mentais próximos aos modelos conceituais da escola, em consequência, não compreendem com solidez o que se tentou ensinar. Evidencia-se a lacuna existente entre os modelos mentais dos estudantes e os modelos conceituais que a escola pretende que eles aprendam.

Sublinha Hestenes (2006) que modelos mentais representam estados do mundo como concebido, não como percebido! Conhecer uma coisa é formar um modelo mental para essa coisa. A partir disso, o autor considera que qualquer tipo de raciocínio ocorre pela inferência da estrutura de modelos mentais. Desse modo, ele distingue pelo menos sete tipos de raciocínios por modelagem mental: o abdutivo, que completa ou estende um modelo mental inicial, frequentemente guiado por um quadro de sentido onde o modelo é embutido; o dedutivo, que extrai a subestrutura de um modelo mental; o indutivo, que combina modelos mentais por experiência; o analógico, que interpreta ou compara modelos mentais; o metafórico, que introduz a estrutura em um modelo mental; o de síntese, que constrói um modelo mental, possivelmente por analogia ou misturando outros modelos; e o de análise, que recorta ou elabora a estrutura implícita em um modelo mental. Conhecer esses tipos de raciocínios por modelagem mental pode auxiliar na tarefa docente. 
Apresentamos até aqui alguns tópicos considerados relevantes sobre modelos mentais. Mas ainda é preciso entender como a TM propõe agir deliberadamente sobre eles, isso requer entender outro conceito: o de modelo conceitual.

\section{Modelos conceituais}

Enquanto os modelos mentais são subjetivos, internos, privados e representados por meio de sistemas mentais, os modelos conceituais pretendem ser objetivos, externos, públicos e representados por meio de sistemas simbólicos (semióticos). Para entender sobre a epistemologia de modelos conceituais na perspectiva da TM, necessitamos entender o conceito de modelo (em sentido amplo), o conceito de estrutura e o conceito de sistema.

Argumenta Hestenes (2006) que os termos sistema e modelo têm sido onipresentes na ciência. No entanto, esses termos são usados muitas vezes de maneira informal e seus significados são bastante variáveis, por isso, é preciso defini-los tão claramente quanto possível. Assim, um sistema foi definido na TM como "[...] um conjunto de objetos relacionados" (HESTENES, 2006, p. 41, grifos do autor e tradução nossa). Os sistemas podem ser de quaisquer tipos, dependendo da natureza dos objetos que 0 compõe. Em casos mais simples, um sistema em si pode ser entendido como um objeto; em casos mais complexos, os objetos dos quais o sistema é composto podem ser considerados subsistemas. Em um sistema conceitual os objetos são conceitos, em um sistema material os objetos são coisas materiais. Dependendo das relações e das propriedades atribuídas aos objetos, um sistema material pode ser classificado como físico, químico ou biológico.

Raramente, em atividades de modelagem, deixa-se claro aos aprendizes sobre 0 significado do termo sistema. É necessário esclarecer sobre especificidades desse termo, pois ele é latente nos procedimentos realizados. Ao compreender que um sistema é constituído por um conjunto de objetos que se relacionam, é provável que o aprendiz comece a observar sua realidade com o intuito de identificar objetos constituintes; bem como identificar como esses objetos estão relacionados. Em outras palavras, ao se explicitar o conceito de sistema ao sujeito, ele tende a se perguntar: como tal sistema é composto? Essa questão epistemológica possibilita evidenciar objetos do sistema, suas interações intrínsecas e extrínsecas, o que nos leva à noção de estrutura.

A estrutura de um sistema é definida na TM como "[...] o conjunto de relações entre objetos em um sistema" (HESTENES, 2006, p. 42, tradução nossa). As relações especificam a composição geométrica, causal e temporal. Comenta ainda que os cientistas estudam sistemas do mundo real não pela observação direta, mas pela construção de modelos para interpretar as observações e representar os objetos na mente.

No início de suas proposições, Hestenes (1987) definiu um modelo em sentido amplo como sendo um objeto substituto, uma representação conceitual de uma coisa real. Alguns anos depois, essa definição seria revista: "um modelo é a representação da estrutura de um dado sistema" (HESTENES, 2010, p. 17, grifos do autor, tradução nossa). $O$ fato é que é comum identificarmos um modelo com sua representação externa 
em um registro de palavras, símbolos ou figuras. Porém, os mecanismos internos para codificação da estrutura do modelo não são nada triviais, exigem um sistema de regras e de convenções semiocognitivas (a maioria tácita). A TM procura iluminar essa codificação.

Em resumo, um sistema pode ser entendido como um conjunto de objetos que mantêm certas relações entre si. A estrutura de um sistema pode ser compreendida como um conjunto dessas relações. Já um modelo (em sentido amplo) pode ser entendido como uma representação para estrutura de um dado sistema. Por exemplo, se o objetivo é modelar a melhor maneira para se organizar os carros em um estacionamento, então o sistema é o próprio estacionamento e os carros são os objetos do sistema. Mas se queremos modelar o fluxo de calor na sala de aula, então o sistema é a sala de aula e os objetos podem ser as paredes, carteiras, lâmpadas, pessoas. O tipo de sistema é que define o tipo de objeto, consequentemente, o tipo de relações para a construção do modelo.

Contudo, os carros em um estacionamento mudam de posição, especificam mudança na geometria do sistema. As pessoas em sala de aula também especificam mudanças na geometria do sistema. Enquanto os objetos em si (de um certo ponto de vista) permanecem imutáveis, suas relações podem mudar facilmente com o tempo, apontam mudanças na estrutura do sistema como um todo. Assim, a estrutura de um sistema é função das relações entre os objetos. O conceito de estrutura é fundamental na TM porque não modelamos os próprios objetos pertencentes a um sistema, mas suas relações. Não estamos interessados em modelar efetivamente os carros em um estacionamento, mas encontrar relações que levem à melhor maneira de estacioná-los. De igual modo, não estamos interessados em modelar efetivamente as pessoas em uma sala de aula, mas relações que nos levem a entender como elas afetam o fluxo de calor. E o melhor instrumento para isso é a construção de um modelo.

D. Hestenes usa o termo forma simbólica para a tríade de elementos que definem um modelo em sentido amplo: representação, estrutura e referente.

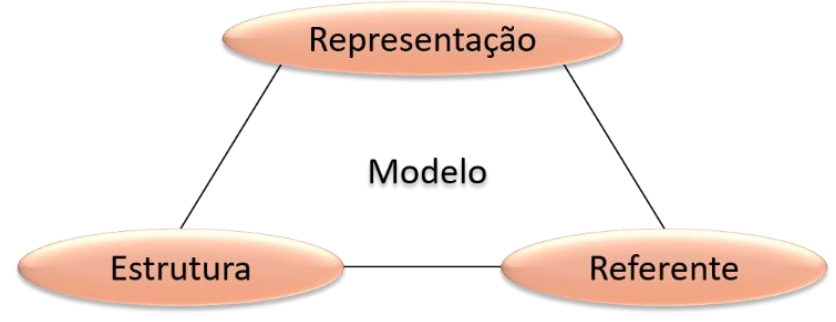

Figura 2 - Forma simbólica de um modelo (Fonte: HESTENES, 2010, p. 18, tradução nossa).

A figura anterior delineia que, qualquer tipo de modelo, necessariamente, possui estes três elementos: representação, estrutura e referente. A representação de um modelo codifica sua estrutura a partir de regras tácitas. No caso de modelos matemáticos, tal codificação é feita por meio de linguagem matemática que possibilita fazer inferências sobre o referente. 
A partir dessas considerações gerais sobre sistema, estrutura e modelo, é possível especificar discussões sobre o que seria um modelo conceitual. Primeiramente, Hestenes (2006, p. 42) definiu um modelo conceitual "[...] como uma representação da estrutura de um sistema material, que pode ser real ou imaginário" (grifos do autor e tradução nossa). Mas essa ideia ainda seria revisada. Nesse sentido, visando inserir pressupostos advindos da linguística cognitiva no arcabouço da TM, D. Hestenes propõe uma definição para modelo conceitual a partir da definição de conceito. Com base na linguística cognitiva, um conceito é entendido na TM como um par \{forma, significado\} representado por um símbolo (ou sistema simbólico).

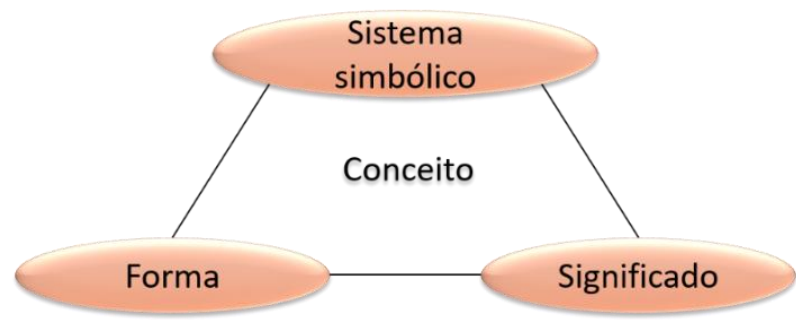

Figura 3 - Tríade Conceitual (Fonte: HESTENES, 2006, p. 45, tradução nossa).

Para ser compartilhado publicamente, por ser naturalmente abstrato, um conceito deve ser representado por meio de uma escritura simbólica (visível). O sistema simbólico escolhido para representar um conceito é responsável por atribuir-lhe morfologia (forma) e fisiologia (significado). Por exemplo, ao escolher representar o conceito de força por meio do registro algébrico $\boldsymbol{F}=m \boldsymbol{a}$, estamos atribuindo-Ihe a forma algébrica e o significado da Segunda Lei de Newton, sendo que o significado e a forma de um conceito são alimentados por um modelo mental prototípico (HESTENES, 2006).

A partir da definição de conceito dada anteriormente, Hestenes redefine a ideia de modelo conceitual, a saber: "um modelo conceitual é agora definido como um conceito (ou construto) com a condição adicional que a estrutura de seu referente ser codificada por uma construção simbólica, ou figura, ou alguma outra inscrição" (HESTENES, 2006, p. 46, grifos do autor e tradução nossa). Desse modo, tal como um conceito, um modelo conceitual pode ser caracterizado por uma tríade: representação, estrutura e referente.

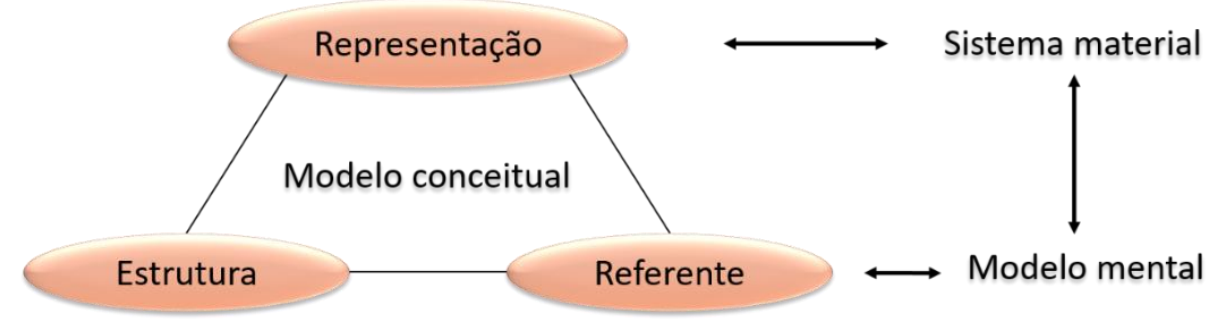

Figura 4 - Modelo conceitual (Fonte: HESTENES, 2006, p. 46, tradução nossa).

Conforme ilustra a figura precedente, a ideia de modelo conceitual é uma extensão da ideia de conceito. Um modelo conceitual é gerado quando se codifica a estrutura de um modelo mental (o referente) por meio de uma representação simbólica ou sistema semiótico. Ou seja, diferentemente de um conceito, um modelo conceitual é visível, 
concreto, pode ser manipulado de alguma maneira a partir transformações de registros de representação. Ademais, o referente de um modelo conceitual é sempre um modelo mental. Ou seja, devemos distinguir dois tipos de representação para um modelo conceitual: uma representação externa (objetiva) em termos de sistemas simbólicos (visíveis) e uma representação interna (mental ou subjetiva) na mente do sujeito modelador (HESTENES, 1987). Desse modo, Hestenes faz uma primeira aproximação entre modelos conceituais e modelos mentais.

Para fortalecer essa aproximação, obedecendo ao Princípio das Formas Universas, D. Hestenes propõe cinco tipos de estruturas básicas para caracterizar os modelos conceituais:

\section{Quadro 2: Estruturas universais de um modelo conceitual.}

\begin{tabular}{|c|c|}
\hline Estruturas & Características \\
\hline Sistêmica & $\begin{array}{l}\text { Sua representação especifica (a) composição do sistema, (b) ligações entre as partes (objetos } \\
\text { individuais), (c) ligações para agentes externos (objetos no ambiente). Uma representação } \\
\text { diagramática é geralmente a melhor (com os objetos representados por nós e ligações } \\
\text { representadas por linhas conectadas) porque permite uma imagem holística da estrutura } \\
\text { como um todo. Exemplos: diagramas de circuitos elétricos, gráficos de setores, árvores de } \\
\text { famílias. }\end{array}$ \\
\hline Geométrica & $\begin{array}{l}\text { Especifica (a) configuração (relações geométricas entre as partes), (b) localização (posição } \\
\text { com respeito a um quadro de referência). }\end{array}$ \\
\hline Objeto & $\begin{array}{l}\text { Propriedades intrínsecas das partes. Por exemplo, massa e carga se os objetos forem coisas } \\
\text { materiais, ou funções se os objetos forem agentes com comportamento complexo. Os objetos } \\
\text { podem eles mesmos serem sistemas (tais como átomos compostos de elétrons e núcleos), } \\
\text { mas suas estruturas internas não são representadas no modelo, embora possam ser } \\
\text { refletidas nas propriedades atribuídas. }\end{array}$ \\
\hline Interação & $\begin{array}{l}\text { Propriedades das ligações (tipicamente interações causais) geralmente representadas como } \\
\text { relações binárias sobre partes de objetos. Exemplos de interações: forças (mudanças de } \\
\text { momentum, transporte de matéria de qualquer forma, mudança de informação). }\end{array}$ \\
\hline Temporal & $\begin{array}{l}\text { Mudança temporal no estado do sistema. Mudança na posição (movimento) é o tipo mais } \\
\text { fundamental de mudança, pois ela permite medida básica de tempo. A teoria da medida } \\
\text { especifica como quantificar as propriedades de um sistema dentro de propriedades variáveis. } \\
\text { O estado de um sistema é um conjunto de valores para suas propriedades variáveis (em um } \\
\text { dado tempo). Mudança temporal pode ser representada descritivamente (como em gráficos), } \\
\text { ou dinamicamente (por equações de movimento ou leis de conservação). }\end{array}$ \\
\hline
\end{tabular}

Fonte: Hestenes (2010, p. 18-19).

As estruturas caracterizadas no Quadro 2 para modelos conceituais são as mesmas propostas pelo autor para modelos mentais. Ou seja, tanto modelos conceituais quanto modelos mentais possuem cinco tipos de estruturas básicas: sistêmica, geométrica, do objeto/descritiva, de interação e temporal.

Além disso, para ir mais a fundo na ideia de modelo conceitual, D. Hestenes utiliza um dos principais pressupostos da linguística cognitiva, a saber, "(...) os referentes da linguagem são modelos mentais em vez de objetos concretos no mundo externo (HESTENES, 2010, p. 19, grifos do autor e tradução nossa). Isto quer dizer que nenhuma prática de modelagem tem como alvo aspectos do mundo real em si, mas os verdadeiros alvos são modelos mentais que formamos para raciocinar sobre o que se considera realidade. Nas aulas de física, por exemplo, não modelamos a queda de objetos em si, mas um modelo mental que formamos para entender essa situação cotidiana! 
Essas ousadas reflexões levaram Hestenes a refinar cognitivamente a definição de modelo conceitual dada anteriormente, posto que: "um modelo conceitual é uma representação da estrutura de um modelo mental" (HESTENES, 2010, p. 19, grifos do autor e tradução nossa). Em outras palavras, um modelo conceitual seria uma inscrição simbólica coordenada a um modelo mental (HESTENES, 2015b). Nessa atualização, o autor estreita ainda mais a relação entre modelos conceituais e modelos mentais.

Argumenta ainda Hestenes (2006) que modelos mentais são construções privadas na mente de um indivíduo. Eles podem ser convertidos a modelos conceituais pela codificação estrutural por meio de inscrições simbólicas (registros semióticos) que ativam e correspondem a modelos mentais de outros indivíduos.

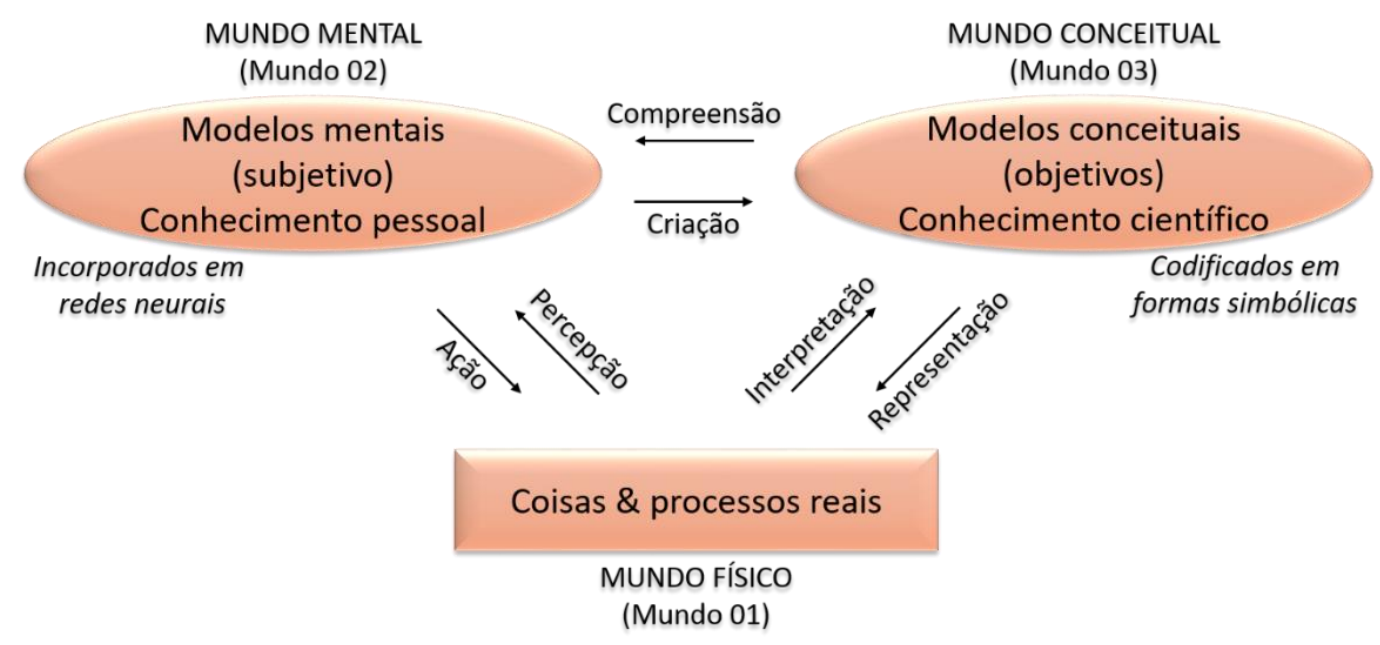

Figura 5 - Modelos mentais vs. modelos conceituais (Fonte: HESTENES, 2006, p. 44, tradução nossa).

Essa figura ilustra uma relação entre modelos mentais, mundo físico e modelos conceituais. Modelos mentais, subjetivos e incorporados em redes neurais, possibilitam tanto a percepção quanto a ação sobre processos e coisas da realidade; além disso, participam ativamente da criação e da compreensão de modelos conceituais, estes, objetivos e codificados em inscrições simbólicas, permitindo, portanto, a interpretação e a representação de processos e coisas do mundo percebido como real. Na prática efetiva de sala de aula, as ideias alinhavadas acima são materializadas por meio da Ml.

\section{Modeling Instruction}

$\mathrm{Na}$ seção anterior, vimos que a TM considera que a cognição humana é basicamente construção e manipulação de modelos mentais. Todavia, modelos mentais formados a partir da experiência diária geralmente são incompatíveis com modelos conceituais ensinados na escola. Assim, um dos problemas principais da aprendizagem em ciências e em matemática é relacionar modelos mentais com modelos conceituais. Como isso pode ser feito? O objetivo nesta seção é discutir sobre características principais da MI. 
Jackson, Dukerich e Hestenes (2008) comentam que o nome Modeling Instruction expressa a ênfase na construção e na aplicação de modelos conceituais de fenômenos físicos como aspecto central da aprendizagem e do fazer ciências. Os estudantes são engajados no discurso e no debate compartilhado de conteúdos e de técnicas científicas. Sua essência é corrigir muitas fraquezas do método tradicional, inclusive a fragmentação do conhecimento, a passividade dos estudantes e a persistência de crenças ingênuas sobre o mundo físico. O professor procura desenvolver habilidades nos estudantes para que deem significado a experiências físicas, para que compreendam afirmações científicas, para que articulem coerentemente suas próprias opiniões e as defendam com argumentos convincentes, além de avaliar evidências para apoiar e justificar suas crenças. As orientações são norteadas por uma agenda pedagógica bem definida. Os conteúdos são organizados sobre modelos conceituais como unidades coerentes da estrutura do conhecimento ao invés de blocos de conteúdo fragmentados. Os estudantes engajam-se colaborativamente na construção e no uso de modelos para descrever, explicar, predizer, planejar e controlar fenômenos físicos.

Eric Brewe (2008) descreve a Ml em um curso de física universitário norteamericano enfatizando as fases de desenvolvimento, de aplicação, de adaptação, de extensão e de revisão de modelos em um esforço para materializar o papel destes em sala de aula. $O$ autor comenta que a didática possibilitou a organização efetiva do conhecimento consistente com a prática aceita cientificamente. Comenta ainda que o conteúdo do curso foi organizado em um pequeno número de modelos gerais que puderam ser aplicados em uma ampla classe de situações. Isso resultou em pelo menos dois benefícios: primeiro, a organização curricular levou a uma expertise em modelagem (estudantes peritos); segundo, os discentes estudaram um pequeno número de modelos gerais como um corpo de conhecimento coerente. Assevera ainda E. Brewe que, tanto a pedagogia quanto a organização de conteúdo são centrais na $\mathrm{Ml}$, no entanto, o mecanismo de construção de modelos não é muito conhecido, significando que os estudantes examinam o fenômeno para construí-los e que eles (os modelos) existem em um nível estrutural das leis da física e acima de um nível conceitual dos alunos. Por fim, reflete o autor, embora haja muitos benefícios para a adoção da MI nas aulas de física, também existem alguns impedimentos. Um deles é a falta de compreensão do papel dos modelos conceituais no ensino. Outro problema é que o currículo de modelagem aplicado ao ensino médio é diferente do universitário. A pesquisa de Eric Brewe é interessante quando aborda diversas questões no ensino superior de física e que, ressalvadas as peculiaridades educacionais, podem servir de orientação ao contexto brasileiro.

Para Hestenes (2010), a principal característica da Ml consiste em ser uma abordagem instrucional investigativa centrada no estudante e orientada pelo professor. Ela focaliza a compreensão de um sistema ou de um processo físico concreto. $O$ professor guia sutilmente o processo investigativo com questões, com sugestões e com desafios; ele apresenta equipamentos, termos padrões, convenções e ferramentas representacionais quando necessário. Os estudantes logo compreendem que o objetivo da investigação é formular e avaliar um modelo conceitual bem definido do sistema em questão. O mecanismo orientador principal é o discurso de modelagem, o que significa que o professor enquadra todo o discurso de sala de aula em termos de modelos e de modelagem. $O$ objetivo é sensibilizar os estudantes para a estrutura do conhecimento 
científico, tanto em aspectos procedimentais quanto declarativos. Dois métodos são fundamentais na MI: o ciclo de modelagem e a gestão do discurso de modelagem.

\section{Ciclos de modelagem}

As implicações da MI para a prática efetiva de sala de aula possibilitaram, ressalta Hestenes (2010), a elaboração de atividades investigativas organizadas em ciclos de modelagem. De maneira geral, um ciclo de modelagem pode ser planejado em dois momentos: o desenvolvimento do modelo e a aplicação (ou implementação) do modelo.

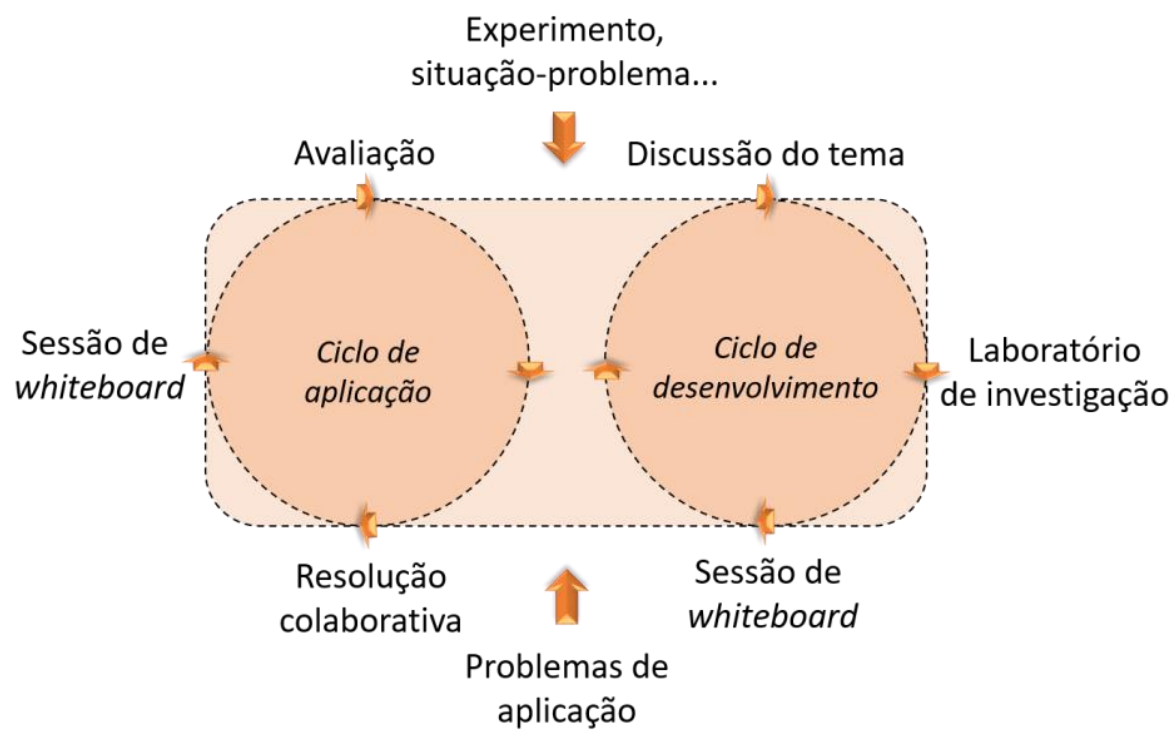

Figura 6 - Estrutura de um ciclo de modelagem (Fonte: Elaboração nossa, 2017).

Chamamos a atenção que um ciclo de modelagem é composto por dois subciclos. Um que inicia com a discussão de um tema que servirá para contextualizar o desenvolvimento de um modelo conceitual, outro que inicia com a discussão de problemas que servirão para refinar compreensões sobre a estrutura epistêmica do modelo desenvolvido. Evidentemente, o esquema precedente pode ser modificado para atender especificidades pedagógicas de determinado contexto educacional. Em última análise, ele pretende uma vista geral sobre a dinâmica de um ciclo de modelagem na perspectiva da TM, o que pode ser importante para o planejamento de práticas efetivas de sala de aula no contexto brasileiro.

O desenvolvimento do modelo pode ser planejado em três ações: 1) discussão do tema; 2) laboratório de investigação e 3) sessão de whiteboard². O tema a ser modelado pode ser de diferentes naturezas: um experimento de laboratório ou experimento de baixo custo financeiro, uma situação-problema, uma simulação computacional etc. Após a escolha do tema, é necessário problematizá-lo com o objetivo principal de evidenciar variáveis e constantes, ou seja, grandezas científicas que se inter-relacionam e que comporão o futuro modelo conceitual. Hestenes (1987) argumenta que a discussão de um tema é orientada por alguma teoria científica, pois é a teoria que especifica quais tipos de

2 Whiteboards são pequenos quadros brancos medindo aproximadamente $70 \mathrm{~cm} \times 70 \mathrm{~cm}$, servem para representar modelos conceituais no decorrer do movimento argumentativo dos estudantes. 
objetos e de propriedades podem ser modelados e quais tipos de modelos podem ser desenvolvidos. Algumas perguntas podem nortear a discussão do tema: quais características estão variando no sistema? Quais características permanecem constantes? Quais características afetam outras características? Hestenes (2010) propõe que na fase de descrição sejam evidenciados os cinco tipos de estruturas universais dos modelos: estrutura sistêmica, estrutura geométrica, estrutura descritiva, estrutura de interação e estrutura temporal. Por fim, define-se uma questão de modelagem a ser investigada por toda a classe.

Definida a questão de modelagem, a classe é organizada em grupos colaborativos para dar início ao laboratório de investigação. A palavra laboratório não deve ser entendida como um ambiente fechado cheio de equipamentos técnicos, mas um momento em que serão planejadas e realizadas atividades que envolverão a observação de campo, a experimentação, a prática de determinada arte ou habilidade, enfim, os erros e os acertos. O trabalho com grupos é importante para gerar uma comunidade de aprendizagem (DESBIEN, 2002). O normal é que cada grupo contenha de três a cinco componentes. A questão de modelagem orienta a produção e representação de dados qualitativos e dados quantitativos pelos próprios estudantes. Isso envolve discussões para o planejamento de procedimentos necessários e o levantamento de informações em fontes diversas. O produto do laboratório de investigação é um conjunto de informações que devem ser logicamente fazendo-se uso de múltiplas inscrições simbólicas (verbal escrita, algébrica, diagramática, gráfica), de modo a propor respostas à questão de modelagem. Os modelos conceituais são então registrados nos whiteboards, preferencialmente, utilizando-se marcadores de texto de diferentes cores. Desse modo, cada whiteboard organiza um modelo (ou parte dele) que será defendido e discutido coletivamente pela classe.

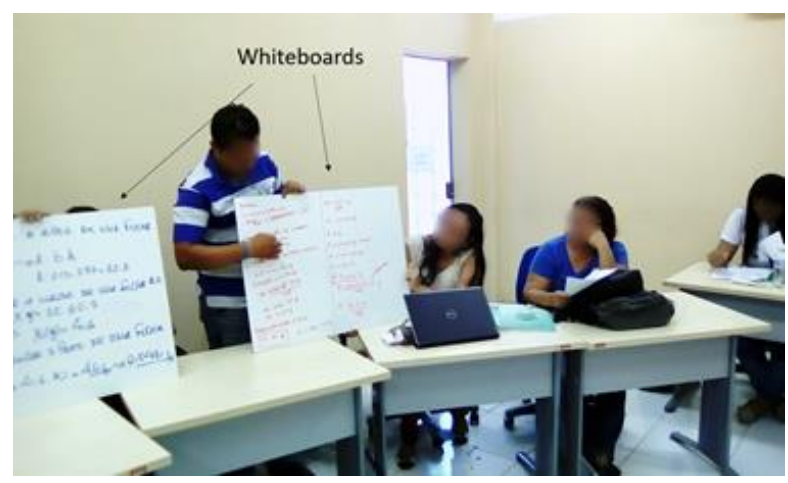

Figura 7 - Sessão de whiteboard (Fonte: Captação nossa, 2017).

$\mathrm{Na}$ primeira sessão de whiteboard, as equipes apresentam seus modelos conceituais e justificam procedimentos e pensamentos. O professor assume a importante tarefa de orientar os discursos dos estudantes de maneira a fazer com que insiram suas justificativas em teorias e em leis científicas, sendo que os modelos conceituais servem de âncoras para tal inserção. Isso possibilita que os estudantes associem seus modelos mentais às inscrições simbólicas ao interpretá-las cientificamente em meio a explicações, justificativas e previsões. Esse processo de argumentação científica com apoio expresso de múltiplas representações pode favorecer à reformulação de modelos mentais incoerentes. Conforme os modelos vão sendo compartilhados pelos grupos, as 
discussões entre os pontos convergentes e os pontos divergentes permitem compreensão comum sobre a estrutura epistêmica dos modelos conceituais.

Conforme a Figura 6, a aplicação do modelo pode ser planejada em três ações: 4) resolução colaborativa; 5) sessão de whiteboard e 6) avaliação. Esse momento inicia com a discussão de problemas cuidadosamente escolhidos com foco no interesse pedagógico da disciplina e que suscitem a estrutura epistêmica do modelo conceitual recém desenvolvido pelos grupos. Importante ressaltar que não se trata de uma lista longa de exercícios, mas preza-se pela qualidade de poucos problemas a serem resolvidos de maneira colaborativa para alcançar os assuntos de interesse pedagógico.

Os problemas de aplicação são importantes porque os estudantes aprofundam compreensões ao ramificarem o modelo conceitual na análise de aspectos matemáticos e de interpretações científicas. Hestenes (1987) sublinha que a ramificação é um processo principalmente matemático e é importante para se trabalhar propriedades e implicações especiais do modelo conceitual. Por exemplo, equações de movimento são resolvidas para determinar trajetórias com várias condições iniciais, resultados são representados e analisados analiticamente e graficamente. Quando existe necessidade e condições apropriadas, o modelo ramificado é validado pela avaliação empírica, em alguns casos, a validação envolve refinados experimentos de laboratório.

As soluções dos problemas de aplicação geram relatórios escritos. Neles, as equipes organizam suas respostas ao sistematizarem os procedimentos realizados e apresentarem discussões críticas. Em nova sessão de whiteboard, cada equipe defende suas resoluções e justificam procedimentos e pensamentos. Novamente, o professor assume a importante tarefa de orientar os discursos dos estudantes de modo a fazer com que insiram suas justificativas em teorias e leis científicas. Conforme as soluções dos grupos vão sendo compartilhadas e refinadas, a estrutura epistêmica do modelo conceitual ganha novos significados que favorecem aplicações em situações diversas.

A avaliação é formativa durante todo o ciclo de modelagem, mas o professor pode checar de alguma maneira a aprendizagem individual dos estudantes e decidir em resolver outros problemas de aplicação ou iniciar um novo ciclo para o estudo de outro campo conceitual. Nesse sentido, temos utilizado portfólios de aprendizagem e alcançado bons resultados avaliativos. Ambrósio (2013) argumenta que o portfólio consiste de uma coleção de trabalhos realizados pelos estudantes que permite acompanhar seus desenvolvimentos por meio de diferentes formas de análise, permite ainda avaliar, executar e apresentar produções desencadeadas de ações de ensino e de aprendizagem desenvolvidas num determinado tempo-espaço. Nesse processo, o estudante guarda suas produções, produções essas que vão mostrar pistas, evidências, vestígios dos conceitos, fatos, procedimentos, atitudes desenvolvidas durante um tempo mediado pelo docente.

No decorrer de um ciclo de modelagem, é essencial que o professor possua habilidades para gestão argumentativa em sala de aula, é o chamado discurso de modelagem. 


\section{Discurso de modelagem}

Uma pesquisa de doutorado desenvolvida por Dwain Desbien (2002) mostrou que somente desenvolver e aplicar modelos conceituais sem que o professor promova situações argumentativas em que os estudantes possam discutir sobre conhecimentos factuais, procedimentais, epistemológicos resulta em ganho de aprendizagem no $\mathrm{FCl}^{3}$ considerado baixo. Por outro lado, a presença de processos argumentativos em ambiente de ensino sem que ocorra construção e aplicação de modelos conceituais resulta em ganho de aprendizagem igualmente baixo no $\mathrm{FCl}$. Em vista disso, o autor chegou à conclusão de que é imprescindível que o professor possua alguns conhecimentos específicos para gerir situações argumentativas em ciclos de modelagem.

Tais conhecimentos fazem parte do que se tem chamado de discurso de modelagem. O objetivo do discurso de modelagem é motivar os estudantes na criação de interações dialógicas em sala de aula. Consultando a literatura, é possível identificar dois tipos básicos: a gestão socrática (referente ao modo como o filósofo Sócrates aplicava sua dialética) e a gestão desbieniana (idealizada por Dwain Desbien).
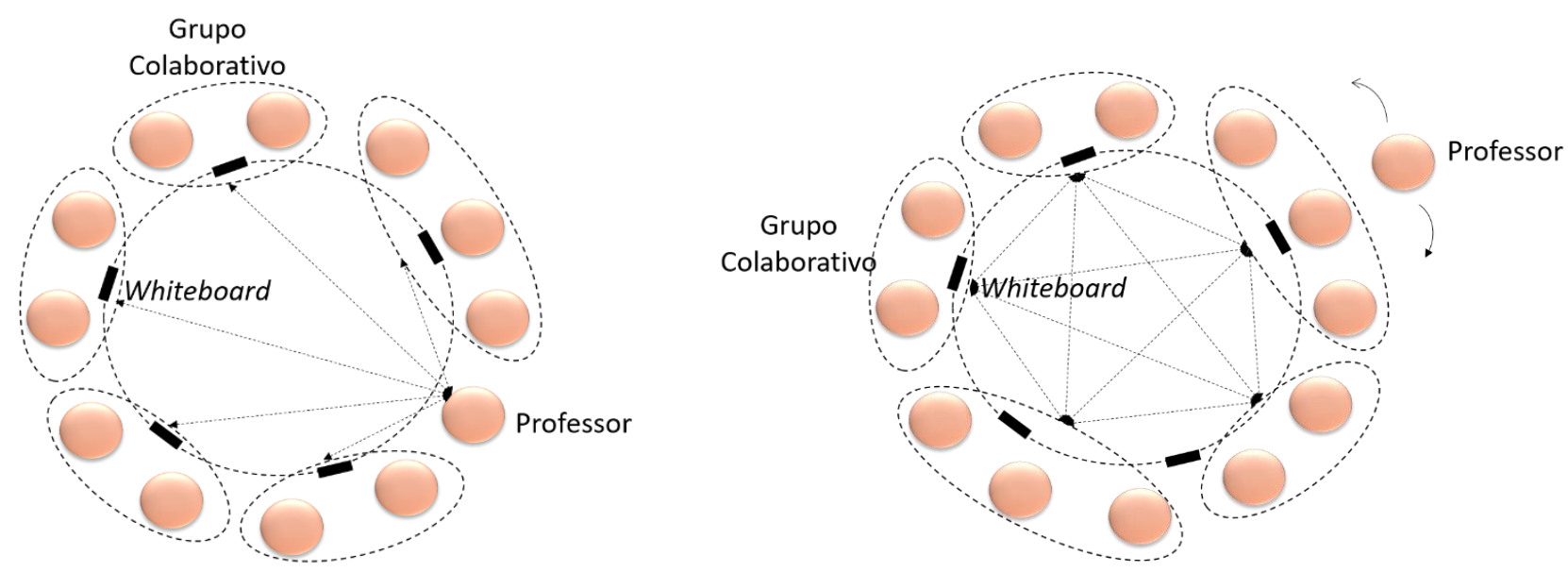

Figura 8 - Gestão socrática (esquerda) e gestão desbieniana (direita) (Fonte: Elaboração nossa, 2017).

$\mathrm{Na}$ gestão socrática, o professor induz situações argumentativas ao questionar diretamente pequenos grupos colaborativos ou mesmo a classe toda, o discurso principal geralmente é do tipo professor $\leftrightarrow$ estudantes. Nesse tipo de discurso, o professor assume a função de conduzir argumentações por meio de questionamentos e de problematizações, ele assume a função de lançar diretamente perguntas para os grupos ou para toda a classe. Embora seja o mais comum em ciclos de modelagem, nesse tipo de discurso a interação intergrupos precisa ser continuamente estimulada. $O$ professor toma parte como ator principal do discurso ao direcionar questionamentos aos grupos, que raramente interagem entre si.

Na extremidade esquerda da Figura 8, ilustramos a organização da classe sugerida para o discurso socrático. Nota-se que o professor faz parte da circunferência juntamente com os demais estudantes. Cada grupo colaborativo dispõe seu whiteboard de modo a

\footnotetext{
${ }^{3}$ Force Concept Invetory, um teste de múltipla escolha mundialmente utilizado contendo trinta questões cujo
} objetivo é avaliar o ganho de aprendizagem em mecânica newtoniana. 
ser visível para os demais da classe. A função do professor é lançar diretamente questionamentos que levem os estudantes à argumentação científica.

Por outro lado, na gestão desbieniana, o professor "orbita" o processo discursivo, que focaliza principalmente as interações estudante $\leftrightarrow$ estudante. Nesse caso, as perguntas partem dos próprios grupos em direção a outros grupos ou em direção a toda a classe, ou seja, os próprios grupos são responsáveis por induzir argumentações científicas por meio de questionamentos. Isso não quer dizer que o professor é passivo no andamento do discurso intergrupos, ao contrário, ele é ativo no sentido de "semear" ideias em determinados grupos, ideias que serão transformadas em questionamentos geradores de argumentação. Além disso, o professor usa seu conhecimento disciplinar para oferecer ferramentas de modelagem, atividades, materiais de apoio, terminologias científicas. Nesse caso, o professor desloca-se da posição de ator principal do discurso para a posição de ator coadjuvante ao interagir indiretamente com comunidade de aprendizagem. Isso faz com que o discurso fique focalizado nos próprios grupos colaborativos, que aumentam o nível interacional e formam uma comunidade de aprendizagem que se fortalece com o tempo.

$\mathrm{Na}$ extremidade direita da Figura 8, ilustramos o discurso desbieniano em que a classe também é organizada em formato semelhante ao de circunferência. Porém, diferentemente do discurso socrático, o professor não ocupa lugar nela. Ele a orbita para semear ideias em certos grupos colaborativos que as lançarão no grupo maior. Isso faz com que ocorra maior interação entre os grupos e menor dependência do professor no desenvolvimento dos movimentos argumentativos.

\section{Considerações finais}

Nosso objetivo neste paper foi apresentar a Teoria da Modelagem de David Hestenes como possibilidade teórica ao ensino brasileiro de ciências e de matemática. A questão de pesquisa foi investigar como são os fundamentos principais dessa teoria e como ela vem sendo aplicada em sala de aula. Esperamos ter alcançado, ao menos parcialmente, nossos objetivos, posto que passaremos discutir sobre algumas implicações da TM para a pesquisa na educação científica e matemática.

A primeira implicação diz respeito uso dos whiteboards como favorecedores da argumentação dos discentes. Vimos que os pequenos quadros brancos são fundamentais para argumentação científica porque contribuem para elaboração e socialização de modelos conceituais de maneira compartilhada entre os estudantes. Podem ser manuseados com facilidade e servem como plataforma para registrar recursivamente os modelos, consequentemente, reformular inconscientemente modelos mentais. Assim, eles são importantes porque favorecem a coordenação de modelos conceituais com os modelos mentais. Uma pergunta que surge é: como os whiteboards podem contribuir para a estruturação de argumentos coerentes dos estudantes? Essa questão é importante para entender potencialidades da argumentação com uso dos whiteboards em relação à argumentação convencional, ou seja, sem a utilização desse recurso de representação e de comunicação. 
Outra implicação a ser colocada é quanto à possibilidade de desenvolver letramento científico com uso da MI. Vimos que a TM surgiu da preocupação de D. Hestenes na aprendizagem conceitual em física. Da mesma maneira, a aplicação da MI tem sido feita visando-se preponderantemente ao conteúdo conceitual, o que gerou 0 desenvolvimento do $\mathrm{FCl}$, cujo objetivo é avaliar crenças dos estudantes sobre conceitos da mecânica newtoniana (HESTENES, WELLS, SWACKHAMER, 1992). Concordamos sobre a importância de que os estudantes adquiriram certa bagagem conceitual em ciências e em matemática. Entretanto, pensamos que seja igualmente importante a aquisição de outras dimensões da educação científica e matemática, como o conhecimento procedimental e o conhecimento epistemológico. Isso é importante para que eles possam analisar criticamente problemas do cotidiano que envolvam ciência, tecnologia, sociedade e meio ambiente visando tomada de decisão para melhorar suas vidas e de suas comunidades. Nesse sentido é que nossa agenda de pesquisas procura investigar: como ocorre o desenvolvimento de letramento científico em práticas de $\mathrm{MI}$ no contexto do ensino de física?

Uma terceira implicação, talvez a mais delicada, é quanto à possibilidade de investigar a estrutura dos modelos mentais a partir da estrutura dos modelos conceituais. D. Hestenes propõe que isso possa ser feito com base nos esquemas cognitivos que entram no cenário do espaço mental durante uma tarefa de modelagem conceitual. Por exemplo, ao representar um objeto em queda livre, um estudante mobiliza modelos mentais para representar e comunicar seus pensamentos por meio de sistemas simbólicos. Na via de mão dupla, esses mesmos sistemas simbólicos poderiam dar pistas sobre a qualidade dos modelos mentais subjacentes em suas produções. Nesse sentido, algumas perguntas surgem para esquentar as discussões: quais sistemas simbólicos são comumente utilizados pelos estudantes para estruturar modelos conceituais? $O$ que determina a utilização de um sistema em detrimento de outro? Como o Princípio das Formas Universais pode promover um link entre modelos mentais e modelos conceituais?

Esperamos apenas ter lançado as sementes sobre o terreno da TM com a intenção que outros pesquisadores possam germinar e, quem sabe, possamos juntos colher bons frutos para o ensino brasileiro de ciências e de matemática.

\section{Referências}

AMBRÓSIO, M. O uso do portfólio no ensino superior. Rio de Janeiro: Vozes, 2013 (Livro digital).

AMTA. AMERICAN MODELING TEACHERS ASSOCIATION. 2017. Disponível em: $<$ http://modelinginstruction.org/>. Acesso em 10 mai 2017.

BREWE, E. Modeling theory applied: Modeling Instruction in introductory physics. American Journal of Physics, v. 76, n. 12, p. 1155-1160, 2008. Disponível em: http://dx.doi.org/10.1119/1.2983148. Acesso em 03 fev 2017.

BREWE, E.; KRAMER, L.; O'BRIEN, G. Modeling instruction: positive attitudinal shifts in introductory physics measured with class. Physics Education Research. v. 05, n. 013102, 2009.

Disponível

em: 
https://journals.aps.org/prper/abstract/10.1103/PhysRevSTPER.5.013102. Acesso em 23 mai 2017.

DESBIEN, D. M. Modeling discourse management compared to other classroom management styles in university physic. 2002. Doctor of Education - Arizona State University Arizona. Disponível em: http://perusersguide.org/items/detail.cfm?ID=7287. Acesso em 10 mai 2017.

DITMORE, D, A. Effect of modeling instruction on concept knowledge among ninth grade physics students. 2016. Doctor of Education - Walden University. Disponível em: htp://scholarworks.waldenu.edu/dissertations. Acesso em 10 mai 2017.

DYE, J. et all. The impact of modeling instruction within the inverted curriculum on student achievement in science. Electronic Journal of Science Education, v. 17, n. 02, 2013. Disponível em: http://ejse.southwestern.edu/article/view/11231. Acesso em 10 mai 2017.

HALLOUN, I. A.; HESTENES. D. O. Modeling Instruction in mechanics. Am. J. Phys. v. 55, n. 05, 1987, p. 455-462. Disponível em: http://aapt.scitation.org/doi/10.1119/1.15130. Acesso em 02 fev 2017.

HEIDEMANN, L. A; ARAUJO, I. S.; VEIT, E. A. Ciclos de modelagem: uma proposta para integrar atividades baseadas em simulações computacionais e atividades experimentais no ensino de física. Cad. Bras. Ens. Fís., v. 29, n. Especial 2, 2012, p. 965-1007. Disponível em: https://periodicos.ufsc.br/index.php/fisica/article/view/21757941.2012v29nesp2p965. Acesso em 02 fev 2017.

HESTENES, D. Modeling theory and modeling instruction for STEM education. 2015a. Disponível em: https://secure.hbcse.tifr.res.in/epi6/papers/Reviewtalks/epiSTEME6_ReviewTalk_David\%20Hestenes.pdf. Acesso em 07 ago. 2016.

Disponível

Conceptual Modeling in physics, mathematics and cognitive science. 2015b. http://semioticon.com/semiotix/2015/11/conceptual-modeling-in-physics-mathematics-andcognitive-science/. Acesso em 07 ago. 2016.

. Modeling theory for math and science education. In: LESH, Richard et al. Modeling student's mathematical modeling competencies. New York: Springer, 2010. Disponível em: https://link.springer.com/chapter/10.1007\%2F978-1-4419-0561-1_3. Acesso em 02 fev 2017.

. Notes for a modeling theory of science, cognition and instruction. In: Proceedings.... Amsterdam: University of Amsterdam, 2006, p. 34- 65. Disponível em: http://modeling.asu.edu/R\&E/Notes_on_Modeling_Theory.pdf. Acesso em 21 mar 2017.

HESTENES, D., WELLS, M., e SWACKHAMER, G. Force concept inventory. The Physics Teacher, v. 30, p. 141-158, 1992.

JACKSON, J.; DUKERICH, L.; HESTENES, D. Modeling instruction: an effective model for science education. Science Educator, v. 17, n. 01, p. 10-17, 2008. Disponível em: http://files.eric.ed.gov/fulltext/EJ851867.pdf. Acesso em 02 fev 2017. 
JOHNSON-LAIRD, N. P. The history of mental models. 2005. Disponível em: http://mentalmodels.princeton.edu/papers/2005HistoryMentalModels.pdf. Acesso em 17 mar 2016.

KILPELA, D. W. The effects of modeling instruction on student learning of a newtonian force concept. 2013. (Master of Science, Michigan Technological University). Disponível em: http://www.mtu.edu/cls/education/pdfs/reports/Kilpela_Report_2013.pdf. Acesso em 10 maio 2015.

MALHEIROS, B. T. Metodologia da pesquisa em educação. Rio de Janeiro: LTC, 2011.

MAZUR, E. Peer instruction: a revolução da aprendizagem ativa. Tradução Anatólio Laschuk. Porto Alegre: Penso, 2015 (Livro Eletrônico).

SOUZA, E. S. R. A formação de modelos mentais na sala de aula. Exitus, v. 03, n. 01, p. 169-184, 2013.

SOUZA, E S. R.; ROZAL, E. F. Instrução por modelagem de David Hestenes: uma proposta de ciclo de modelagem temática e discussões sobre alfabetização científica. Amazônia Revista de Educação em Ciências e Matemática, v. 12, n. 24, p. 99-115, 2016.

WELLS, M. Modeling instruction in high school physics. 1987. Doctor of Education Arizona State University. Disponível em: http://modeling.asu.edu/modeling-HS.html. Acesso em 10 mai 2017.

WELLS, M.; HESTENES, D.; SWACKHAMER, G. A modeling method for high school physics instruction. 1995. American Journal of Physics. Disponível em: http://dx.doi.org/10.1119/1.17849. Acesso em 24 de jul., 2016.

Agradecemos à Coordenação de Aperfeiçoamento de Pessoal de Nível Superior (CAPES) pela bolsa Prodoutoral-UFOPA.

Submissão: 08/08/2016

Aceite: 05/09/2017 